LETTERS

If you have a burning desire to respond to a paper published in JECH, why not make use of our "rapid response" option?

Log on to our web site (www.jech.com), find the paper that interests you, and send your response via email by clicking on the "eletters" option in the box at the top right hand corner.

Providing it isn't libellous or obscene, it will be posted within seven days.

You can retrieve it by clicking on "read eletters" on our homepage.

The editors will decide as before whether to also publish it in a future paper issue.

\section{Using ACME (Automatic Classification of Medical Entry) software to monitor and improve the quality of cause of death statistics}

Various methods have been used to evaluate the quality of cause of death statistics. Traditionally, necropsy findings were deemed as the gold standard to evaluate the accuracy of cause of death certification. However because of the biased selection of necropsy cases and the decreasing necropsy rate, fewer and fewer evaluation studies have used necropsy findings as the standard. ${ }^{3}$ Another commonly used standard to evaluate the quality of death certification is the consensus of a panel of physicians reviewing all available information related to the deceased. ${ }^{4}$ Most of the studies using this method were the byproducts of large cohort studies or randomised clinical trials. These studies wanted to assure that the end point was not biased. The shortcomings of using physician review as the standard were time consuming, costly, not applicable in a large scale and routinely. ${ }^{5}$

As more and more disease specific registries and hospital medical records were computerised, more and more investigators began to use these datasets as the standard to evaluate the quality of cause of death statistics. The merits of this method were time saving, less costly, applicable in large scale and routinely. Several population based studies used computer linkage of cause of death file and hospital discharge file to compare the underlying cause of death (UC) and discharge diagnosis. ${ }^{7-10}$ Almost "every" deceased were medically attended in developed countries, these studies could thus evaluate the quality of "every" death certification. Johansson and Westerling attempted to develop a systematic and routine monitoring mechanism at the national level that can detect the poor death certification "before" the publication of mortality data. ${ }^{10}$ Unlike the previous post hoc studies, through this monitoring system, once the poor quality of death certification was identified, they could immediately query the certifier to modify the death certification. Thus a better quality of cause of death statistics could be attained immediately after the evaluation.

One limitation of this method was that the definition of hospital discharge main diagnosis and the UC do not coincide. Johansson and Westerling further innovatively used ACME (Automatic Classification of Medical Entry) software to solve this limitation, which rendered the monitoring system more robust. ${ }^{11}$ Many people might not be very familiar with the ACME. In the following, I firstly introduce what ACME is and how it works. Then, I point out some limitations of ACME and the possibility of improvement.

\section{What is ACME?}

To improve the comparability of cause of death statistics among different countries, International Selection Rules for selecting the UC was set by World Health Organisation. ${ }^{12}$ Nevertheless, the rules leave room for interpretation, which resulted in differences in the selection of UC across countries. ${ }^{13}{ }^{14} \mathrm{To}$ tackle the problem of inconsistency among coders within and across countries, in the late 1960s and early 1970s the US National Center for Health Statistics (NCHS) developed the ACME computer system to standardise the production mortality statistics.

ACME uses information based on not only the International Classification of Diseases (ICD) codes for each reported condition on the death certificate, but also their actual location on the death certificate. The computer program then applies each international selection rule in sequence to these codes, resulting in a code for a temporary underlying cause (TUC). This TUC code is then subjected to each international modification rule in sequence, finally yielding assignment of a single UC code. ${ }^{15}{ }^{16}$ The core of ACME is the Decision Tables, which provide specific relations between one code and another to establish whether the causal sequence is acceptable, highly improbable, or acceptable as a consequence of Rule 3, or whether other modification rules are needed. ${ }^{17}$ ACME has been used in many countries and broad adoption would certainly improve the comparability of mortality across countries. ${ }^{18}$ One important feature of ACME is that the logics of selecting the UC for each death certificate could be visualised, which could be used for education and training purposes. The following three examples with different complexity in layouts of diagnoses on death certificates were used to illustrate how ACME processed.

\section{Example 1}

I (a) Acute myocardiac infarction (I219)
(b) Hypertension (I10)
(c) Diabetes (E149)

\section{ACME process messages of example 1}

01 I219/110/E149

02 Is I2 19 due to E149? YES

03 Is I10 due to E149? YES

04 Select Initial TUC $=$ E149-General Principle

05 ACME UC: E149
Example 2

I (a) Congestive heart failure (I509)

(b) Cerebral infarction (I639), endocarditis (I38)

(c) Liver cirrhosis (K746)

(d) Hypertension (I10)

II Chronic obstructive pulmonary disease (J449),

Oral cancer (C069)

ACME process messages of example 2

01 I509/1639 I38/K746/I10*J449 C069

02 Is I509 due to I10? YES

03 Is I639 due to I10? YES

04 Is I38 due to Il0? YES

05 Is K746 due to I10? NO

06 No TUC by General Principle-Apply Rule 1 07 Is I509 due to I639? YES

08 Is I639 due to K746? NO

09 Select TUC $=$ I639-Rule 1

10 ACME UC: 1639

Example 3

I (a) Congestive heart failure (I509)

(b) Cerebral infarction (I639), endocarditis (I38)

(c) Liver cirrhosis (K746), uremia (N19), Diabetes (E149)

(d) Hypertension (I10)

II Chronic obstructive pulmonary disease (J449), Oral cancer (C069)

ACME process messages of example 3

01 I509/I639 I38/K746 N19 E149/I10*J449 C069

02 Is I509 due to I10? YES

03 Is I639 due to I10? YES

04 Is 138 due to I10? YES

05 Is K746 due to I10? NO

06 No TUC by General Principle-Apply Rule 1

07 Is I509 due to I639? YES

08 Is I639 due to K746? NO

09 Is I639 due to N19? YES

10 Is N19 due to I10? YES

11 Select TUC $=$ I10 - Rule 1

12 Linkage due to position condition I10 I509 I1 10 MAYBE

13 Linkage with mention of preference I10 I639 YES

14 Linkage due to position preference I10 I38 YES

15 Linkage with mention of combination I10 N19 I120 YES

16 Is I509 due to I639? YES

17 Is I639 due to K746? NO

18 Is I639 due to N19? YES

19 Select TUC $=$ N19-Rule 1

20 Select TUC $=$ I1 20 -Rule $\mathrm{C}$ linkage

21 ACME UC: I120 


\section{Limitations of ACME}

Though ACME has been deemed as the de facto international standard for interpreting ICD selection rules, it is not without problems. First limitation was that there were many "MAYBE" causal relations in the decision tables, which needed manual assignments for the UC. Examples were listed as follow:

- Is K746 (liver cirrhosis) due to A419 (sepsis)? MAYBE

- Is K746 (liver cirrhosis) due to B169 (hepatitis B infection)? MAYBE

- Is I698 (sequels of stroke) due to El49 (diabetes)? MAYBE

- Is J449 (chronic obstructive pulmonary disease) due to I64 (stroke)? MAYBE

- Is J189 (pneumonia) direct sequel of I509? MAYBE

- Is R54 (senility) and I509 (heart failure) combined as R54? MAYBE

If different countries had different decisions for above "MAYBE" cases, this became another source of artefact undermining the comparability of mortality data across countries.

Another limitation, ironically this is in fact the strength of ACME, was the rigid adherence to the selection rules that resulted in the over-coding of mechanism of death (MOD). The MOD is a physiological derangement or a biochemical disturbance produced by a cause of death. Examples include various arrhythmias, renal failure, cardiopulmonary failure, sepsis, and hypovolaemic shock. The cause of death, on the other hand, is a distinct entity, and is aetiologically specific. Examples include cerebrovascular infarction, lung cancer, diabetes mellitus, and alcoholic liver cirrhosis. Because of their lack of aetiological specificity, MOD should not appear on death certificates ${ }^{19-21}$ Nevertheless, because medical treatment is often aimed at modifying or ameliorating mechanisms rather than causes, thereby physicians still filled many MODs on death certificate. This poor certification behaviour was fueled by high frequency of incorrect layout of diagnoses on the death certificates. Previous studies revealed that it was very common for physicians to enter two or more diagnoses in the same line in death certificate. ${ }^{22-24}$ Examples were:

- I (a) Uraemia, diabetes

- I (a) Heart failure, liver cancer

- I (a) Hepatic failure, ischaemic heart disease

Another common certification error was the reverse layout of causal relations. For example, hypovolaemic shock (HS) was due to oesophageal varices bleeding (EVB) and EVB due to liver cirrhosis (LC). A correct layout should put HS in line (a), EVB in line (b), and LC in line (c), nevertheless it was not very uncommon that the certifier might put HS in line (c), EVB in line (b), and LC in line (a). Other examples were:

- I (a) Acute myocardial infarction

(b) Pneumonia

(c) Sepsis

- I (a) Stroke

(b) Urinary tract infection

(c) Sepsis

According to international selection rule 2 (for first three examples) and general principle (for last two examples), ACME would select MOD-that is, uraemia, heart failure, hepatic failure, and sepsis as the UC for above examples. Most people will agree that these results were obviously not the original intents of the certifiers. MOD could not provide useful information for prevention.

Luckily many of the above mentioned problems might be solved in Mortality Reference Group (MRG), which was set up by the World Health Organisation with the mandate to issue authoritative instructions on the interpretation of the ICD coding rules and guidelines. The NCHS have pledged themselves to implement the decision of the MRG in ACME decision tables. It is hoped that the modified Decision Tables will be more acceptable to people in most countries.

T H Lu

Department of Public Health, Chung Shan Medical University, Taichung, Taiwan 402; robertlu@ms1.hinet.net

\section{References}

1 Alderson MR. Mortality, morbidity and health statistics. Basingstoke, UK: Macmillan, 1988.

2 Maudsley G, Williams EMI. 'Inaccuracy' in death certification - where are we now? J Public Health Med 1996; 18:59-66.

3 Moriyama IM. Problems in measurement of accuracy of cause-of-death statistics. Am J Public Health 1989;79: 1349-50.

4 Start RD, Bury JB, Strachan AG, et al. Evaluating the reliability of causes of death in published clinical research. BM 1997;314:271.

5 Bangdiwala SI, Cohn R, Hazard C, et al. Comparisons of cause of death verification methods and costs in the lipid research clinics program mortality follow-up study. Control Clin Trials 1989;10:167-87.

6 Lu TH, Chang HY, Hwu CM, et al. Comparison of official coders versus physician panel in assignment of underlying cause of death. J Formos Med Assoc

2001;100:365-9.

7 Gittelsohn AM, Senning J. Studies on the reliability of vital and health records: I. Comparison of cause of death and hospital record diagnoses. Am J Public Health 1979;69:680-9.

8 Goldacre MJ. Cause-specific mortality: understanding uncertain tips of the iceberg. J Epidemiol Community Health 1993:47:491-6.

9 O'Hara D, Hart W, Robinson M, et al. Mortality soon after discharge from a major teaching hospital: linking mortality and morbidity. J Qual Clin Pract 1996;16:39-48.

10 Johansson LA, Westerling R. Comparing Swedish hospital discharge records with death certificates: implications for mortality statistics. Int J Epidemiol 2000;29:495-502.

11 Johansson LA, Westerling R. Comparing hospital discharge records with death certificates: can the differences be explained? $J$ Epidemiol Community Health 2002:56:301-8.

12 World Health Organisation. International statistical classification of diseases and related health problems, tenth revision, volume 2 . Geneva: WHO, 1993.

13 Jougla E, Pavillon G, Rossollin F, et al. Improvement of the quality and comparability of causes-of-death statistics inside the European Community. Revue Epidemiol Sante Publique 1998;46:447-56.

14 Lu TH, Lee MC, Chou MC. Accuracy of cause of death coding in Taiwan: types of miscoding and effects on mortality statistics. Int $J$ Epidemiol 2000;29:336-43.

15 Israel RA. Automation of mortality data coding and processing in the United States of America. World Health Stat $Q$ 1990;43:259-62.

16 Birch D. Automatic coding of causes of death. Popul Trends 1993;73:1-3.

17 National Center for Health Statistics. Instruction manual part 2c: ICD-10 ACME decision tables for classifying underlying causes of death. Hyattsville, MD: US Department of Health and Human Services, Public Health Services, 2001.

18 National Center for Health Statistics. Homepage-Initiative and other activities. Available at http://www.cdc.gov/nchs/ about/otheract/ice/automort/automort.htm. (Accessed 15 October 2002.)

19 National Center for Health Statistics. Physicians' handbook on medical certification of death. Hyattsvelle, MD: US Department of Health and Human Services, 1987.

20 Kircher T, Anderson RE. Cause of death: proper completion of the death certificate. JAMA 1987:258:349-52.

21 Hanzlick R. The medical cause of death manual: instructions for writing cause of death statements for deaths due to natural causes. Northfield: College of American Pathologists, 1994.

22 Leadbeatter S. Semantics of death certification. J $R$ Coll Phys London 1986;20:129-32.

23 Slater DN. Certifying the cause of death: an audit of wording inaccuracies. J Clin Pathol 1993;46:232-4

24 Lu TH, Shou WY, Shih TP, et al. Factors associated with errors in death certificate completion: a national study in Taiwan. J Clin Epidemiol 2001;54:232-8.

\section{Seasonality of live birth sex ratio in south western Siberia, Russia, 1959-2001}

Seasonality of sex ratio of live births (SR: male births divided by total births) has been reported in Europe, North America, Brazil, and Australia. However, no uniform pattern is seen. ${ }^{1}$ Moreover, the magnitude of any observed seasonal variation varies from population to population with marked variation in Japan $^{2}$ to minor variation in Germany ${ }^{3}$ to none in south western Finland, Scotland, Costa Rica, and Hausa, Africa. The population of Novosibirsk region was 2767938 in 1988. Siberian climate exhibits considerable seasonal temperature changes. In Novosibirsk over the period 1951-1980, the average difference in mean monthly air temperature between January (the coldest month, $-18.8^{\circ} \mathrm{C}$ ) and July (the warmest month, $19.0^{\circ} \mathrm{C}$ ) was $37.8^{\circ} \mathrm{C}$. We tested the null hypothesis that there is no seasonal variation in SR in Siberia.

Records of live singleton births were obtained from the Novosibirsk Regional Committee for Statistics. Data by month were obtained for the years 1959-2001, excluding 1961, 1962, and 1988 because of missing data. Seasonal analysis was carried out by Edwards' method. Our analysis was quarterly because of the comparatively small number of births. Linear regression analysis was performed to test for secular trend.

A highly significant seasonal pattern was evident $\left(\chi^{2}=14.4, \mathrm{p}=0.001\right)$ with an amplitude of $1.2 \%$ of the overall mean, a peak in the second quarter $\left(\theta=129^{\circ}\right)$ and a trough in the fourth quarter (fig 1).

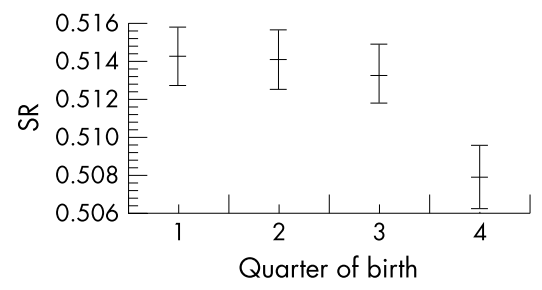

Figure 1 Seasonality of sex ratio at birth in Novosibirsk region, Russia, 1959-2001. SR: male births divided by total births. Values are means and $95 \%$ confidence intervals. 


\section{Key points}

- Male births in Siberia fall sharply in the last quarter of the year.

- This implies reduced male conceptions or reduction in survival of male conceptuses in the first quarter of the year.

- If this effect is temperature related, low temperatures may be implicated through unknown mechanism/s.

- Industrialisation has not reduced male births in Siberia

A negative annual secular trend was found for the period $1971-1980(r=-0.84, \mathrm{p}=0.002)$ which was replaced by the positive trend during the period 1982-1993 $(r=0.78, \mathrm{p}=0.004)$. No difference in mean SRs for the entire period was found between urban (0.513) and rural $(0.513)$ populations.

The decrease in male births in the last quarter equates to fewer male conception nine months previously-that is, in the first quarter. Climatic variations in west Siberia are extreme, with heavy snowfalls in winter. Thawing of snow requires considerable energy, therefore temperatures remain low in spring, and rise sharply from the second half of April. If the observed variation in SR is indeed temperature related, then it would seem that low temperatures either reduce male conceptions or, through unknown mechanism/s, reduce the survival of male conceptuses

Industrialisation has been blamed for declining SRs in industrialised countries ove the past half century. In Siberia, a different pattern is evident in that SR fell and then rose with a turning point in the early 1980s.

\section{Acknowledgement}

We thank Mrs G Bessonova, head of the Department of Novosibirsk Regional Committee for Statistics, for providing the raw data on births.

V N Melnikov

Novosibirsk State University, Novosibirsk, Russia

V Grech

Paediatric Department, St Luke's Hospital, Malta

Correspondence to: $\operatorname{Dr} \bigvee \mathrm{N}$ Melnikov, Novosibirs State University, PO Box 175, Novosibirsk 630060,Russia; mevlanic@.nsk.ru

\section{References}

1 James WH. Interpregnancy intervals, high maternal age and seasonal effects on the human sex ratio. Hum Reprod 1996:11.7-8.

2 Huntington E. Sex, season and climate. In:

Season of birth: its relation to human abilities. New York: Wiley, 1938:192-214.

3 Lerchl A. Seasonality of sex ratio in Germany. Hum Reprod 1998;13:1401-2.

\section{BOOK REVIEWS}

Trauma, war and violence.

\section{Public mental health in} socio-cultural context

Edited by J de Jong. (Pp 454; price not stated). Kluver Academic/Plenum Publishers, New York, 2002. ISBN 0-306-46709-7.

This is a valuable contribution to the scan literature on organising effective public menta health programmes for traumatised refugee or war-torn populations. The book focuses specifically on the public health aspects of complex humanitarian and political emergencies.

The editor opens the book with a long chapter on public mental health, emphasising culturally appropriate models. Especially valuable is the section on the objectives and selection of priorities for training and mental health interventions, with both excellent theoretical and practical aspects.

Nine chapters then follow, describing programmes that are supported by the Transcultural Psychosocial Organisation (TPO), of which Joop de Jong is the director. Each chapter opens with a description of the history and culture of the country and the current problems, which often have received minimal attention by the media, Western populations, and others. The populations described have been chronically traumatised by war, torture, hunger, rape, displacement, and often, wholesale destruction of their society and culture.

The authors of each chapter describe their attempts to assess and improve the mental and medical health of refugee populations, with mixed or no support from local governments. How they use local healers or other supports to build a network of interventions is a key element of each programme. Their frustrations and failures are also articulated.

The chapters vary in length and quantity. For example, "The Cambodian experience" is 64 pages long, and only the most dogged reader will persist to the end. Some of the chapters would have benefited by better editing by an English speaker.

In summary, the opening chapter of the book is an important contribution to the literature on public health and on traumatic stress. The chapters that follow will be of especial interest to those planning to set up similar programmes.

Elspeth Cameron Ritchie ITC, MC, USA and Program Director, Menta Health Policy and Women's Issues, OASD/HA

\section{International co-operation in health}

Edited by $M$ McKee, $\mathrm{P}$ Garner, $\mathrm{R}$ Stott. (Pp 217; price not stated). Oxford University Press, 2001. ISBN 0-192-63198-5.

International co-operation in health focuses on transboundary public health issues and ex- amines the influence of global factors on human health. Historically, the earliest examples arose from changes in the natural environment such as climate changes during the last ice age. Other global events are less obvious but also had important implications (positive and negative) for human health, such as the widespread migration of populations, the enormous effects of international trade, war and civil disorder, or even genetic modified food on human health.

Discussing these global influences, authors of several chapters describe the formal organisations and alliances that are developing to fill the gap between the globalised world and national governments. Suggestions for collaboration predominantly focus on UN structures, for instance, the WHO's efforts in tobacco control, multidisciplinary groups working on global changes coordinated through the UN Environmental programme, and the work of the WTO. Other suggestions focus more on regional (EU) surveillance and prevention networks, responding to challenges posed by infectious diseases.

We may question where the analysis takes us? Discussing these global factors, it becomes clear that globalisation is an extremely complex phenomenon. Although the effects on people may be clear, we still face many challenges when trying to quantify these consequences. The concluding chapter revisits some of the threats that are posed to the public health by globalisation and explores some of the opportunities it offers, in particular, how health professionals can come together to promote global public health. It looks at what health professionals can do to tackle these threats, highlighting principles for actions that encourage collective thinking, mitigating against isolationism and nationalism in confronting problems of society and environment. Finding an answer as to what health professionals can do, the editors advocate that health professionals need to be interested in the changes happening around them; need to look beyond their own national interest as public health problems and solutions have to be considered a global context. More concrete, they propose several valuable suggestions to find common solutions including undertaking research, communicating the information to the public in a clear and comprehensible way, and, most importantly, using this information to lobby for change.

André den Exter, Herbert Hermans

Erasmus University Rotterdam, Netherlands

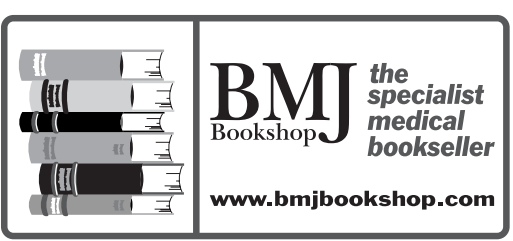

